18 years or older who fulfilled the clinical and epidemiological Health Ministry criteria for case definition of CF were included in the study, from April to December 2016.

Results: From 732 patients included, $83.1 \%$ were women. The mean age was 54.1 ( \pm 13.4 ) years; $92.4 \%$ lived in urban area and $58.6 \%$ had only primary education. The most common comorbidities were hypertension (43.8\%), hyperlipidemia $(25.3 \%)$ and diabetes mellitus (13.7\%). Prior rheumatologic disease was observed in $16.4 \%$ patients, being the most frequent rheumatoid arthritis $(32.5 \%)$, osteoarthritis $(32.5 \%)$ and spondyloarthritis $(11.7 \%)$. Arthralgia was the most frequent symptom referred by all patients; fever and fatigue were also common manifestations, being referred by $95.3 \%$ and $87.1 \%$ of patients, respectively. Arthritis occurred in $84.3 \%$. The most frequent joint pattern involvement was polyarticular $(67.8 \%)$ and the additive $(84.0 \%)$. At the first appointment with the rheumatologist, $75.9 \%$ had been or were under corticosteroid use, with the average dose of $15.4 \mathrm{mg}( \pm 8.7)$ of prednisone or equivalent; was observed an median of 8 painfull joints (IQR 4-21) and arthritis was found in $73.6 \%$ patients, with an median of 2 swollen joints (IQR $0-5)$. The median score of patient global assessment at the time of the initial evaluation was 6 (IQR 4-8) using a 10 points visual analogue scale. After resting stiffness was referred by $86.0 \%$, with $58.4 \%$ of these longer than 30 minutes. The most commonly prescribed medications were corticosteroids (58.3\%) and hydroxychloroquine $(59.1 \%)$. The serological tests for $\mathrm{CHIKV}$ were positive for $\lg \mathrm{M}$ in $97.1 \%$ and for $\lg \mathrm{G}$ in $71.7 \%$ of patients.

Conclusions: This is the first descriptive study of a cohort Brazilian patients with $\mathrm{CF}$, with an expressive number of patients when compared to those described in the literature. Most of the features of patients in our cohort were similar to the results described in studies/cohorts published.

Disclosure of Interest: None declared

DOI: 10.1136/annrheumdis-2017-eular.4537

\section{SAT0558 CONCORDANCE BETWEEN CLINICAL-EPIDEMIOLOGICAL CRITERIA AND CHIKUNGUNYA FEVER SEROLOGY}

C.D.L. Marques ${ }^{1,1,1}$, C.A. Andrade ${ }^{1}$, M.S. Luna ${ }^{1}$, P.R. Santos ${ }^{1}$, A.T. Dantas ${ }^{1}$, A. Ranzolin ${ }^{2}$, N.G. Cavalcanti ${ }^{1}$, R.S. Gonçalves ${ }^{3}$, L.F. da Rocha Jr ${ }^{4}$, P.R. Melo ${ }^{1}$, R.M. Correia ${ }^{3}$, L.D. Valadares ${ }^{5}$, C.A. da Fonte ${ }^{5}$, M.L.D. Valadares ${ }^{5}$ E. Freire ${ }^{6}$, A.K.G. Melo ${ }^{6}$, M.M. Medeiros ${ }^{7}$, M.C. Bezerra ${ }^{7}$, V.B. Marques ${ }^{7}$, R.A. Océa ${ }^{8}$, A.L. Duarte ${ }^{1} .{ }^{1}$ Internal Medicine, Universidade Federal de Pernambuco; ${ }^{2}$ Rheumatology; ${ }^{3}$ Internal Medicine, Instituto de Medicina Integral Prof. Fernando Figueira; ${ }^{4}$ Rheumatology, Instituto de medicina integral professor Fernandes Figueira; ${ }^{5}$ Rheumatology, Hospital Getulio Vargas, Recife;

${ }^{6}$ Rheumatology, Universidade Federal da Paraíba, João Pessoa;

${ }^{7}$ Rheumatology, Universidade Federal do Ceará, Fortaleza; ${ }^{8}$ Rheumatology, Universidade Federal de Sergipe, Aracaju, Brazil

Background: The first autochthonous reports of Chikungunya fever (CF) in Brasil was confirmed in 2014, and by December 2016, there were 263.980 probable cases of CF, $55.03 \%$ confirmed. According to recommendations of the Ministry of Health $(\mathrm{MH})$ of Brazil, in an established epidemic situation, the diagnosis of CF should be made by appliyng clinical and epidemiological criteria. There is no indication for the serology for Chikungunya virus (CHIKV) in the acute phase, except in atypical cases and complicated clinical situations, which may generate doubts in clinical practice about the correct diagnosis of these patients.

Objectives: The objective of this study was to evaluate the concordance of the clinical and epidemiological criteria with the serology results for CHIKV in a cohort of patients with CF.

Methods: The multicenter cohort CHIKBRASIL from the Northeast of Brazil has enrolled CF patients with joint manifestations since April 2016, using as inclusion criteria the presence of fever and arthralgia/arthritis in a patient residing or who had visited an endemic or epidemic area within 15 days prior to the onset of symptoms. For the present study, we selected patients in which lgM and/or lgG serology was performed, regardless of the results. For the analysis of agreement with the serology, the most characteristic symptoms of CF were used individually (fever, arthritis/arthralgia or exanthema) and three models of association of symptoms were created: (1) fever and arthralgia; (2) fever and arthritis; (3) fever, arthralgia/arthritis, and exanthema. The sensitivity (SENS), specificity (SPEC), positive predictive value (PPV) and negative predictive value (NPV) of the criteria were also assessed, with the serology result considered the gold standard.

Results: A total of 143 patients were evaluated, $119(83.2 \%)$ of which were female, with a mean age of 53.89 years $( \pm 13.5)$; $52.4 \%$ of the cases were in the subacute phase of the disease ( 15 days to 3 months) and $42.7 \%$ were in the chronic phase (over 3 months). The IgM positivity was observed in $95.1 \%$ of cases and $\operatorname{lgG}$ in $71.67 \%$. The concordance rate between the $\operatorname{lgM}$ serology or combined positive serology (IgM or positive $\operatorname{lgG}$ ) was over $80 \%$ for any of the symptoms/symptoms model analyzed, as well as the SENS and PPV of the symptoms/ symptoms model, which was over $95 \%$ in all situations evaluated. The concordance rate for IgG serology ranged from 51.9 to $72.1 \%$. Model 1 presented the highest agreement with the result of positive combined serology.

Conclusions: During an epidemic situation, the use of clinical and epidemiological criteria shows high agreement with the serology result, regardless of the combination of symptoms presented, with high sensitivity and positive predictive value. Disclosure of Interest: None declared

DOI: 10.1136/annrheumdis-2017-eular.6431

\section{SAT0559 SEPTIC ARTHRITIS IN COVENTRY IN THE UK: 5 YEAR DATA}

A. Vivekanantham ${ }^{1}$, A. Kahlon ${ }^{1}$, M. Ali ${ }^{1}$, A. Metcalfe ${ }^{2}$, S. Dubey ${ }^{1}$. ${ }^{1}$ Rheumatology; ${ }^{2}$ Orthopaedics, University Hospitals Coventry and Warwickshire, CV2 2DX, United Kingdom

Background: Septic arthritis (SA) is a serious condition associated with significant morbidity and prolonged hospital stays, posing a large economic burden to healthcare systems. It affects $2-10$ people per 100,000 and there has been a suggestion that the incidence is increasing due to iatrogenic causes [1]. Our local secondary centre, University Hospitals Coventry and Warwickshire NHS Trust (UHCW NHS Trust), provides care to Coventry and Rugby covering an estimated population of 550,000 .

Objectives:

- To investigate the incidence of native joint SA in the adult population in a secondary care hospital in the UK.

- To investigate whether immunosuppression contributes significantly to the burden of SA.

Methods: Patients were retrospectively identified on the basis of the International Classification of Diseases (ICD)-10 coding generated following discharge from hospital for all patients between 2007-11. Exclusion criteria included paediatric patients, diabetic foot, prosthetic joint infections and those who on review were not thought to have SA. The data was analysed using Excel. Formal ethical approval was obtained via the research and development department within the UHCW NHS Trust.

Results: A total of 189 admissions were coded as SA. Of these, 103 were excluded $(n=74$ not thought to have $S A$ on review of the notes, $n=26$ paediatric patients and $n=3$ prosthetic joints). Therefore, there were 86 adult admissions for 64 patients with SA.

The average age of these patients was 53.4 years, with the majority of them being males $(n=43,67.2 \%)$. The majority of patients had co-morbidities $(n=44,65.7 \%)$, with hypertension $(n=10,14.9 \%)$ and type 2 diabetes $(n=10,14.9 \%)$ being the most prevalent. Joint aspirates were performed on $63.2 \%(n=56)$ of admissions and blood cultures on $70.8 \%(n=63)$ of admissions. Staphylococcus aureus was the most commonly cultured microbe in both joint fluid $(46.4 \%, n=13)$ and blood $(42.9 \%, n=3)$. The knee was the commonest joint involved $(n=31,46.3 \%)$. Other commonly affected joints included the small joints of the hands $(n=9,13.4 \%)$ and shoulder/acromioclavicular/sternoclavicular joints $(n=9,13.4 \%)$.

Interestingly, $23(35.9 \%)$ of the patients were immunocompromised. Of these, 4 patients had a diagnosis of rheumatoid arthritis (RA) and were on steroid treatment alone $(n=2)$, or in combination with disease-modifying anti-rheumatic drugs $(n=2)$. A total of 11 patients had a pre-existing rheumatological diagnosis of which RA was the most common condition $(n=6)$. Two of these patients were not on immunosuppressants. The 5-year mortality was significant at $29.7 \%(n=19)$.

Conclusions: Our local data showed the incidence of SA to be approximately 3 per 100,000, which is in keeping with proposed figures. Our cohort highlighted that those with pre-existing co-morbidities or those who were immunocompromised were at greatest risk. An ageing population with multiple co-morbidities means the incidence of SA is set to rise. Greater emphasis therefore needs to be placed on improving awareness and optimising treatment.

References:

[1] Geirsson AJ, Statkevicius S, Vikingsson A. Septic arthritis in Iceland 19902002: increasing incidence due to iatrogenic infections. Ann Rheum Dis. 2008:67:638-43.

Acknowledgements: We would like to thank the PPMO team at UHCW NHS Trust.

Disclosure of Interest: None declared

DOI: 10.1136/annrheumdis-2017-eular.4372

\section{SAT0560 COEXISTENCE OF SEPTIC AND CRYSTAL-INDUCED ARTHRITIS: A DIAGNOSTIC CHALLENGE}

Y. Garcia-Mira, A. Prior-Español, S. Minguez, J. Camins, M. Martínez-Morillo, S. Holgado, A. Olive, J. Cañellas, L. Gifre, L. Mateo. H.UNIVERSITARI Germans Trias I Pujol, Badalona, Spain

Background: Septic arthritis (SA) is a rheumatologic emergency as joint destruction occurs rapidly and can lead to significant morbidity and mortality. Accurate diagnosis can be particularly challenging in patients with underlying inflammatory joint disease. Crystal-induced arthritis (CA) is a risk factor for its appearance. When both conditions appear simultaneously, CA may mask diagnosis of infection and delay the antibiotic treatment.

Objectives: To describe the characteristics of patients with concurrent septic and CA.

Methods: Retrospective analysis of patients with coexistence of septic and CA attended between 1985 and 2015 in a university hospital with a reference area of 850,000 inhabitants. We collect demographic, clinical, laboratory and imaging data as well as patient medical treatment, complications and evolution records. All patients had positive bacterial culture (blood and/or joint fluid) and crystals in synovial fluid.

Results: A total of 123 patients with SA were identified. $20.3 \%(n=25)$ of them had concomitant CA, with mean age of 67 years (SD 14), $17(68 \%)$ males and $8(32 \%)$ females. Risk factors were: diabetes (24\%), diuretic drugs $(24 \%)$, chronic renal failure (16\%) -2 of them undergoing hemodialysis and 4 kidney 